\title{
This is Periodontal Disease
}

\author{
José Ricardo Kina* \\ Periodontics Department, Dom Bosco Higher Education Unit, São Luís, Brazil \\ *Corresponding author: José Ricardo Kina, Periodontics Department, Dom Bosco Higher Education Unit, São Luís, Brazil
}

Submission: 眥 February 27, 2018; Published: 非 April 06, 2018

\section{Editorial}

Periodontal disease one of the most common and prevalent diseases of the oral cavity has been a challenge in its etiology, diagnosis and treatment. The etiology of periodontal disease is multifactorial and it is considered that biofilm plays an essential role in its development and evolution. Because of this, the vast majority of periodontal diseases are treated by controlling the biofilm that accumulates around the teeth. Is this kind of method really enough to establish an effective treatment of periodontal disease? It would be interesting to analyze the evidence of how the periodontal disease is established. In order to induce experimental periodontitis [1], it will always be necessary to introduce a predisposing risk factor which associated with biofilm may initiate periodontal disease (e.g. ligature around a tooth, diabetes mellitus). If the predisposing risk factor is not introduced, it will probably be impossible to establish the onset of periodontal disease. The predisposing risk factors may be of a local and general order supporting bacteria to initiate the mechanisms to establish the onset of periodontal disease. Local predisposing risk factor may act promoting bacterial retention increasing their quantity and quality (i.g. overhanging margins of dental restorations) and/or inducing damages in the gingival sulcus facilitating the pathogenic action of opportunistic bacteria (i.g. food impaction with an open dental contact).

General predisposing risk factors are associated with diseases that affect the inflammatory process of the host altering the response against the opportunistic bacteria of the dental biofilm (i.g. diabetes mellitus). Local predisposing risk factor associated with bacteria may induce localized periodontitis only in the periodontium around the teeth where they assist the bacteria initiating the periodontal disease. General predisposing risk factor when associated with bacteria may induce generalized periodontal disease in periodontal tissues around all teeth since the response of the inflammatory process occurs and is similar in all the teeth that present opportunist bacteria. In some cases the risk factors of local and general order may act together and in association with the bacteria to determine the periodontal disease that in this case promote a profile of generalized destruction. Then why is the periodontal disease treated through an attempt to control bacteria and their products? Would it not be more logical during the treatment of periodontal disease to establishing a greater focus on the control of all the etiologic factors involved in the determination of the disease? Of course, however, why is not applied the concept of controlling all the etiological factors involved in determining the disease to treat periodontitis? The main cause is perhaps because the only common etiological factor for any periodontal disease is bacteria [2].

However, would it be possible to establish a control of the bacteria at a level below the index to establish periodontal disease even with the presence of predisposing risk factors? What would be this bacterial count below the potential of establishing periodontal disease for each individual or for each individual tooth? This would be a major challenge because each individual responds differently to the all etiological factors involved in determining a particular periodontal disease. This means that the etiological factors do not maintain a characteristic of specificity for all individuals; what can cause periodontal disease for some individuals may not cause for other individuals. In addition, the progression of periodontal disease occurs by rapid periods of acute destructive inflammatory process always followed by periods of quiescent chronic inflammation [3]. The quiescent periods of chronic inflammation may be reparative since in any histological section of untreated periodontal disease repair of the insertion of the gingival fibers immediately above the destroyed alveolar bone and below the bottom of the untreated periodontal pocket can be observed. Therefore periodontal disease does not present a destructive characteristic throughout its evolution, it ceases the destructive period enters a quiescent period although all the etiological factors are preserved active and even increase in their quantity and quality after a period of acute destructive inflammation. For example, during periodontal disease, after destruction of alveolar bone and periodontal ligament, the tooth loses part of its support that maintain it stable in its alveolus, however the occlusal force continues the same aiming on a tooth with less periodontal support tissue.

Then other questions about periodontal disease arise: What causes the acute destructive inflammatory phase ceasing? When and why does the acute inflammatory destructive phase restart? Are reliable tests available to diagnose the destructive acute phase of periodontal disease? Is it possible to diagnose the destructive acute phase of periodontal disease? Is the treatment of periodontal 
disease being applied at the right time? Would it be healthier to apply the treatment of periodontal disease in the acute destructive phase or in the chronic reparative phase? Periodontal disease still remains as a great mystery to be unveiled and understood [4]. Only then will be possible to diagnose and prevent periodontal disease efficiently and if the disease occurs, to treat the sequelae of the diseased tissues by restoring them to the pre disease stage. At the moment there is no predictable treatment method for returning the periodontal tissues lost during the disease to the pre-disease stage.

Why is unpredictable to recover diseased periodontal tissues to the pre-disease stage? Until now most of the treatments of the periodontal disease aim to recover the tissues lost during the disease but would not it be better to treat the etiologic factors that initiate the periodontal disease that always presents multifactor etiological characteristics? Treating the sequel of a disease is important but in order to establish the treatment of a disease it will always be vital to establish the elimination or control of all the etiological factors that cause the disease.

\section{References}

1. Kina JR, Kina J, Kina EF, Kina M, Soubhia AM (2008) Presence of bacteria in dentinal tubules. J Appl Oral Sci 16(3): 205-208.

2. Kina JR, Suzuki TY, Kina J, Kina M, Kina EFU (2013) Reparative phase events on periodontal disease progression: interpretation and considerations. Int J Microbiol Res 5(4): 439-444.

3. Kina JR, Suzuki TY, Kina J, Kina M, Kina EF (2016) Non-inflammatory destructive periodontal disease. The Open Dent J 10: 50-57.

4. Polson AM (1994) Periodontal regeneration: Current status and directions. Quintessence, Chicago, USA.

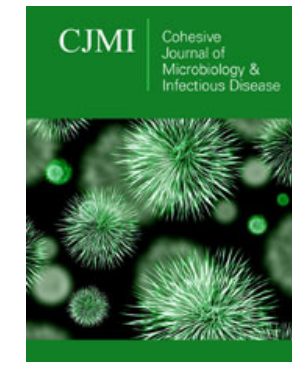

Approaches in Poultry, Dairy \& Veterinary Sciences

\section{Benefits of Publishing with us}

- High-level peer review and editorial services

- Freely accessible online immediately upon publication

- Authors retain the copyright to their work

- Licensing it under a Creative Commons license

- Visibility through different online platforms 